\title{
Determination of stellar, orbital and planetary parameters using complete Monte-Carlo analysis - the case of HAT-P-7b
}

\author{
András Pál ${ }^{1,2}$, Gáspár Á. Bakos ${ }^{1}$, Robert W. Noyes ${ }^{1}$ \\ and Guillermo Torres ${ }^{1}$ \\ ${ }^{1}$ Harvard-Smithsonian Center for Astrophysics, \\ 60 Garden st., Cambridge, MA 02138, USA \\ email: apal@cfa.harvard.edu \\ ${ }^{2}$ Dept. of Astronomy, Eötvös Loránd University, \\ Pázmány Peter stny 1/A, Budapest, 1117, Hungary
}

\begin{abstract}
The recently discovered transiting very hot Jupiter, HAT-P-7b, a planet detected by the telescopes of HATNet, turned out to be among the ones subjected to the highest irradiation from the parent star. In order to best characterize this particular planet, we carried out an analysis based on a complete and simultaneous Monte-Carlo solution using all available data. We included the discovery light curves, partial follow-up light curves, the radial velocity data, and we used the stellar evolution models to infer the stellar properties.

This self-consistent way of modeling provides the most precise estimate of the a posteriori distributions of all of the system parameters of interest, and avoids making assumptions on the values and uncertainties of any of the internally derived variables describing the system. This analysis demonstrates that even partial light curve information can be valuable. This may become very important for future discoveries of planets with longer periods - and therefore longer transit durations - where the chance of observing a full event is small.
\end{abstract}

\section{Introduction}

The transiting extrasolar planet candidate HTR154-011 has been identified by the telescopes of the HATNet (HAT-7, HAT-8 and HAT-6, HAT-9) - see Bakos et al. (2002) for a detailed description about the instrumentation. The reduction of the frames followed the standard frame calibration procedures, followed by star detection, astrometry and aperture photometry which was supported by various detrending algorithms. We search the light curves for box-shaped transit signals using the BLS algorithm (Kovács, Zucker, \& Mazeh 2002): a periodic dip was detected in the light curve of 2MASS $19285935+4758102$. The initial "rejection mode" spectroscopy with CfA Digital Speedometer (Latham 1992) at FLWO eliminated the possibility of an eclipsing binary star system. The analysis of Keck HIRES spectra yielded the radial velocity data, stellar atmospheric parameters and the bisector information (which ruled out a "false positive" hierarchical triple). We also carried out a partial photometry follow-up in the Sloan $z$-band with KeplerCam on the FLWO 1.2m telescope. See Pál et al. (2008) for more details about the observational details and data reduction.

The basics of the characterization of transiting extrasolar planets are the following. The period of the orbital motion can be very accurately determined because of the long timespan of the discovery (HATNet) observations. The mass of the planet is proportional to the amplitude of the RV curve, $K$. The radius of the planet relative to the star 
determines the depth of the transit (which is resulted both by the follow-up light curves and the HATNet data). The inclination of the orbit can be derived from the shape of the transit light curve, i.e. the impact parameter is proportional to the cosine of the inclination. To calculate the absolute mass and radius, we need the same data or the host star too, therefore we need at least one luminosity indicator for a good stellar evolution model. Because of the lack of parallax, this indicator can be $a / R_{\star}$, see Sozzetti et al. (2007). The bottleneck in this case is the partial follow-up, i.e. the shape parameters of the light curve - namely the ingress/egress length relative to the total duration - cannot be derived directly to obtain a reasonable value of $a / R_{\star}$.

\section{System parameters}

To obtain the planetary, stellar and orbital parameters, we used all available data: (i) The HATNet light curves with approximately 16,600 data points (moderate precision with an rms of $7 \mathrm{mmag})$. The HATNet data are affected by the period $P$, epoch $T_{\text {center }}$ (strongly), the planetary radius $p$ (moderately), duration of the transit $T_{\mathrm{dur}}=2\left(\zeta / R_{\star}\right)^{-1}$ (moderately), and the impact parameter $b$ (slightly). (ii) Radial velocity data, high precision relative to the amplitude. The RV data are affected strongly by the period $P$, epoch $T_{\text {center }}$, semi-amplitude $K$, and eccentricity $k=e \cos \omega, h=e \sin \omega$. (iii) The partial follow-up light curve, acquired shortly after the radial velocity measurements. The follow-up photometry data are affected by the period $P$ (slightly), epoch $T_{\text {center }}$ (strongly), planetary radius $p$ (strongly), the duration of the transit $T_{\text {dur }}$ (strongly), and the impact parameter $b$ (strongly).

Because there was only a couple of periods time difference between the radial velocity observations and the partial follow-up photometry, the transit center time could be very well extrapolated, thus the total transit duration can also be accurately obtained. Since there is a strong coupling between the adjustable parameters, the contribution of each parameter in each type of data are different (see above), therefore to obtain a plausible set of system parameters, a joint fit should be performed, incorporating all available data simultaneously.

\section{Monte-Carlo modeling}

The basic system parameters (see previous section) can be determined using a MonteCarlo algorithm (MCMC), resulting an a posteriori distribution of the adjustable parameters. This method also automatically gives the a posteriori distribution of derived quantities, such as $a / R_{\star}$ or the inclination. The luminosity indicator is the density of the star $\rho_{\star}$ which is related to the observable quantities as

$$
\rho_{\star}=\frac{3 \pi}{G P^{2}}\left(\frac{a}{R_{\star}}\right)^{3}-\frac{3 K}{2 P G \sin i}\left(\frac{a}{R_{\star}}\right)^{2} \frac{1}{R_{\star}} \equiv \rho_{0}-\frac{\Sigma_{0}}{R_{\star}},
$$

where both $\rho_{0}$ and $\Sigma_{0}$ are observables, therefore these also have an a posteriori distribution given by the Monte-Carlo fit. The stellar evolution model (Yi et al. 2001) gives the radius of the star as the function of the density, surface temperature $T$ and metallicity $z$, i.e.

$$
R_{\star}=R_{\star}\left(\rho_{\star}, T, z\right) .
$$

Equation (3.1) and equation (3.2) have two unknowns, $R_{\star}$ and $\rho_{\star}$ since $T$ and $z$ are also observables, known from the spectroscopy (and also have a MC distribution from the 


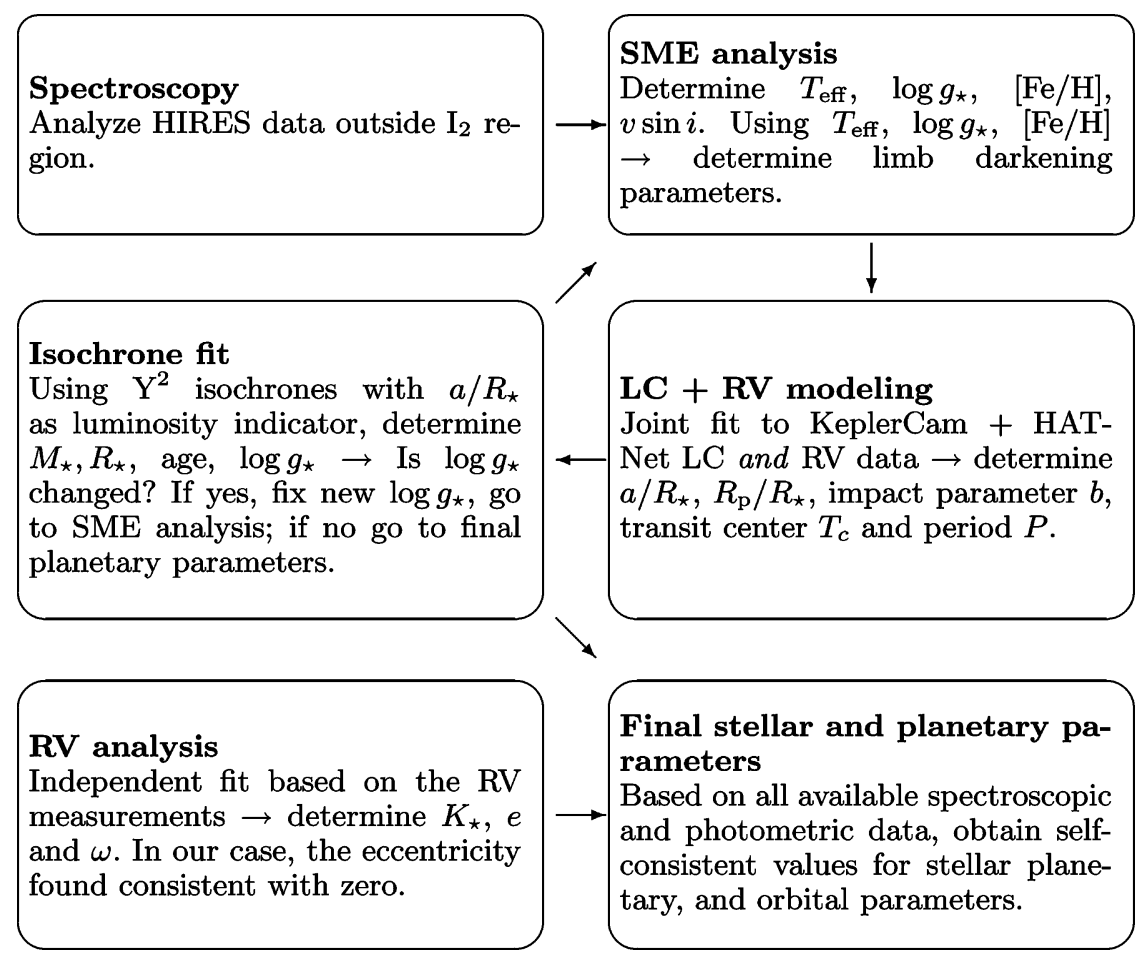

Figure 1. Flowchart of the iterative Monte-Carlo analysis of the stellar, orbital and planetary parameters. The arrows indicate the "data flow" between the subsequent steps. See text for further details.

SME analysis). Therefore, this set of equations can be solved, which yields the a posteriori distribution of the stellar parameters. The combination of the stellar parameters $R_{\star}, M_{\star}$ with the previously fitted quantities results the a posteriori distribution of the planetary parameters in a straightforward way. A sanity check can be done by comparing the resulted stellar surface gravity (obtained directly from $M_{\star}$ and $R_{\star}$ ) with the value provided by the SME. If they differ, the SME analysis has to be redone by fixing the surface gravity and the whole procedure can be done repeatedly until convergence, see flowchart on Fig. 1. We note that the complete MC way of analysis provides the derived parameters independently from the form of calculations, e.g. the a posteriori distribution of the semimajor axis is exactly the same if it is calculated as $a=R_{\star}\left(a / R_{\star}\right)$ or $a=\left[G\left(M_{\star}+M_{\mathrm{p}}\right)\right]^{1 / 3}[P /(2 \pi)]^{2 / 3}$, altough $R_{\star}$ or $a / R_{\star}$ themselves have definitely larger individual uncertainties than the period or $M_{\star}^{1 / 3}$. The final stellar, planetary and orbital parameters for the HAT-P-7(b) system are summarized in Table 1.

\section{Summary}

The outlined method provides a straightforward way for characterizing planetary systems since (i) it omits any kind of intermediate values which otherwise would distort the error estimations, (ii) it automatically provides the uncertainties and correlations of the system parameters, including the stellar, planetary and orbital parameters, (iii) it can utilize even the partial light curves which otherwise would not provide enough information for further steps - these partial follow-up light curves become even more 
Table 1. Stellar, orbital and planetary parameters of the HAT-P-7(b) system.

\begin{tabular}{lcl}
\hline Stellar parameter & Value & Source \\
\hline$T_{\text {eff }}(\mathrm{K})$ & $6350 \pm 80$ & $\mathrm{SME}$ \\
{$[\mathrm{Fe} / \mathrm{H}]$} & $+0.26 \pm 0.08$ & $\mathrm{SME}$ \\
$\log g_{*}(\mathrm{cgs})$ & $4.07 \pm 0.06$ & $\mathrm{Y}^{2}+\mathrm{LC}+\mathrm{SME}$ \\
$v \sin i\left(\mathrm{~km} \mathrm{~s}{ }^{-1}\right)$ & $3.8 \pm 0.5$ & $\mathrm{SME}$ \\
$M_{*}\left(M_{\odot}\right)$ & $1.47 \pm 0.06$ & $\mathrm{Y}^{2}+\mathrm{LC}+\mathrm{SME}$ \\
$R_{*}\left(R_{\odot}\right)$ & $1.84_{-0.11}^{+0.23}$ & $\mathrm{Y}^{2}+\mathrm{LC}+\mathrm{SME}$ \\
$M_{V}($ magnitude $)$ & $3.00 \pm 0.22$ & $\mathrm{Y}^{2}+\mathrm{LC}+\mathrm{SME}$ \\
Distance $(\mathrm{pc})$ & $320_{-40}^{+50}$ & $\mathrm{Y}^{2}+\mathrm{LC}+\mathrm{SME}$ \\
\hline
\end{tabular}

\begin{tabular}{lc}
\hline Orbital/planetary parameter & Value \\
\hline$P$ (days) & $2.204730 \pm 0.000004$ \\
$T_{\text {center }}(\mathrm{BJD})$ & $2,453,790.2593 \pm 0.0010$ \\
$a / R_{*}$ & $4.35_{-0.38}^{+0.28}$ \\
$p \equiv R_{\mathrm{p}} / R_{*}$ & $0.0763 \pm 0.0010$ \\
$b \equiv a \cos i / R_{*}$ & $0.37_{-0.29}^{+0.15}$ \\
$i\left(\right.$ degrees $^{\circ}$ & $85 .^{\circ} 7_{-3.1}^{+3.5}$ \\
\hline$K\left(\mathrm{~m} \mathrm{~s}^{-1}\right)$ & $213.5 \pm 1.9$ \\
$e$ & $0.003 \pm 0.012$ \\
\hline$M_{\mathrm{p}}\left(M_{\mathrm{J}}\right)$ & $1.776_{-0.049}^{+0.077}$ \\
$R_{\mathrm{p}}\left(R_{\mathrm{J}}\right)$ & $1.363_{-0.087}^{+0.195}$ \\
$a(\mathrm{AU})$ & $0.0377 \pm 0.0005$ \\
\hline
\end{tabular}

important when systems with longer periods will be discovered (i.e. the chance to observe a complete transit on a single night will be definitely smaller). The planet HAT-P-7b is also very interesting since (i) it has a very high surface temperature, somewhere between $2200-2700 \mathrm{~K}$, depending on the heat redistribution, (ii) it happens to fall in the field of view of the upcoming Kepler Mission, yielding an opportunity for further continuous observations.

\section{Acknowledgments}

The work of A. P. was supported by the NASA grant NNG04GN74G. Work by G. Á. B. was supported through Hubble Fellowship Grant HST-HF-01170.01-A and by the NSF postdoctoral fellowship grant AST-0702843. Operation of the HATNet is supported by the NASA grants NNG04GN74G and NNX08AF23G.

\section{References}

Bakos, G. Á., Lázár, J., Papp, I., Sári, P., Green, E. M. 2002, PASP, 114, 974

Kovács, G., Zucker, S., \& Mazeh T. 2002, A\&ษA, 391, 36

Latham, D. W. 1992, IAU Coll. 135; ASP Conf. Ser. 32, 110

Pál, A. et al. 2008, ApJ, 680, 1450

Sozzetti, A. et al. 2007, ApJ, 664, 1190

Yi, S. K. et al. 2001, ApJS 136, 417 